was created based on the discussion and all participants of the meeting were surveyed. Replies were compiled to ascertain the current picture of service delivery and educational training.

Results: 25 rheumatology specialist nurses participated in the exercise. Their job experience ranged from one week to 25 years (median 7 years). $21 / 25$ (84\%) ran independent clinics. 16/25 (64\%) were employed at band 6 . Five (20\%) did not feel confident in counseling patients initiating methotrexate. Apart from two, no one had any formal training in delivering DMARD education. 10/25 (40\%) had never been supervised whilst undertaking an educational session for patients. Six (24\%) did not feel confident to teach or supervise their peers.

Conclusions: This is a pioneering survey mapping the training of rheumatology practitioners and nurse specialists to service delivery. This initiative highlights a wide variation in the training structure of a key job provision. There is lack of formal induction programme. Despite most participants running independent clinics and providing DMARD education for years, they confessed to have no formal education and little supervision. Over a fifth did not feel confident in counseling patients commencing methotrexate despite being in the job for a median of 18 months. Though most centres are delivering contemporary services, these are not being used effectively for developing key team members. A quarter of the cohort felt unprepared to impart the skills to peers (median experience 1 year).

In conclusion, there is wide variation in the training of rheumatology nurse specialists. This can potentially have a negative impact on a relatively young workforce. There is a need for improving training standards to help deliver good quality rheumatology professionals of the future.

Disclosure of Interest: None declared

DOI: 10.1136/annrheumdis-2017-eular.2783

\section{THU0747-HPR EFFECT OF DIFFERENT PHYSICAL THERAPY PROGRAMS ON PAIN, STRENGTH AND FUNCTIONAL SITUATIONS ON KNEE OSTEOARTHRITIS}

\section{Ö.E. Günaydın, V. Bayrakcı Tunay. Sports Physiotherapy, Hacettepe University,}

\section{Ankara, Turkey}

Background: Knee Osteoarthritis $(\mathrm{OA})$ is one of the most common causes of disability with an increasing prevalence and incidence with age. Although there are studies in the literature that examine the efficacy of ESWT, Kinesio taping and exercise on knee OA, there is no study to compare these methods with each other.

Objectives: The purpose of this study is to compare the effects of Extracorporeal Shockwave Therapy (ESWT), Kinesio taping and exercise therapy on pain, knee strength and functional situations of patients with knee osteoarthritis (OA)

Methods: Forty eight female patients aged between 50-65 and previously diagnosed with a grade 1-3 (Kellgren-Lawrence scale) knee OA included in this study. Patients were assessed before treatment and after treatment at 6 weeks and at 12 weeks. Visual Analog Scale, (VAS), (ISOMED ${ }^{\circledR} 2000$ D\&R $\mathrm{GmbH}$, Germany) isokinetic device, Timed Up and Go test (TUG) and WOMAC scale were carried out for assessing patients' pain, knee strength and functional situations. After randomising the patients into 3 different groups, the first group received 1 session of ESWT per 6 weeks, the second group received 2 sessions of Kinesio tape per 6 weeks and the third group was prescribed with an exercise program only.

Results: The between-groups results showed a significant improvement for the pain during night, pain during resting, and WOMAC test in the exercise group $(p<0,05)$, while no difference was found for the other measurements $(p>0,05)$. The inter-groups results showed significant improvements in 3 of the groups $(p<0,05)$, while there was no difference in 60 degrees/sec isokinetic quadriceps strength test in the Kinesio taping group and TUG test in the ESWT group.

Conclusions: The results of this study showed that; ESWT, Kinesio tape and exercise therapies are all effective in decreasing pain intensity, improving knee strength and functional status levels of patients with knee OA and can be used as alternative approaches to treat symptoms of knee OA.

\section{References:}

[1] Plotnikoff, R., Karunamuni, N., Lytvyak, E., Penfold, C., Schopflocher, D., Imayama, I. ve diğerleri. (2015) Osteoarthritis prevalence and modifiable factors: a population study. BMC Public Health, 15, 1195.

[2] Sarzi-Puttini, P., Cimmino, M.A., Scarpa, R., Caporali, R., Parazzini, F., Zaninelli, A. ve diğerleri. (2005). Osteoarthritis: an overview of the disease and its treatment strategies [Bildiri].Seminars in arthritis and rheumatism.

[3] De Oliveira Melo, M., Aragão, F.A.,Vaz, M.A. (2013) Neuromuscular electrical stimulation for muscle strengthening in elderly with knee osteoarthritis-a systematic review. Complementary therapies in clinical practice, 19 (1), 27-31.

[4] Zhao, Z., Jing, R., Shi, Z., Zhao, B., Ai, Q.,Xing, G. (2013) Efficacy of extracorporeal shockwave therapy for knee osteoarthritis: a randomized controlled trial. Journal of surgical research, 185 (2), 661-666.

Disclosure of Interest: None declared

DOI: 10.1136/annrheumdis-2017-eular.5437

\section{THU0748-HPR RECOMMENDATIONS ON PHYSICAL THERAPY PRESCRIPTION FOR AXIAL SPONDYLOARTHRITIS IN THE NETHERLANDS}

S. van Weely ${ }^{1}$, F. van der Giesen ${ }^{1}$, N. Lopuhaa ${ }^{2}$, F. van Gaalen ${ }^{3}$, T. Vliet Vlieland ${ }^{1} .{ }^{1}$ Orthopaedics, Rehabilitation and Physical Therapy, Leiden University Medical Center, Leiden; ${ }^{2}$ Dutch Arthritis Foundation, Amsterdam;

${ }^{3}$ Rheumatology, Leiden University Medical Center, Leiden, Netherlands

Background: In national and international guidelines physical therapy, comprising exercise interventions and education, is recommended as a required treatment modality for the optimal treatment of axial spondyloarthritis (axSpA). [1-3] However, specific details regarding referral for physical therapy and optimal content and dose of exercise interventions are lacking. Research showed large variation in the content of exercise therapy in axSpA patients, which reflects suboptimal care [4]

Objectives: To develop practice recommendations on indications for referral, content, dose and safety aspects of exercise therapy for axSpA patients based on scientific evidence, expert opinion and patient values. The ultimate aim is improving the quality of exercise therapy care for people with axSpA.

Methods: The recommendations are based on scientific evidence, expert opinion and patient values and were formulated following a combination of literature review and three expert-group meetings (consisting of patients, rheumatologists, physical and exercise therapists, policy makers, scientists and special interest groups). In three consecutive expert-meetings clinically relevant questions, draft recommendations based on systematic literature reviews, and final recommendations including level of agreement were generated. Lastly, a field consultation among physical and exercise therapists, rheumatologists, scientists and special interest groups will be scheduled and an implementation strategy, comprising of an information intervention and directives, will be developed.

Results: In the first expert-group meeting 18 clinically relevant questions were formulated, on: indication and referral, assessment, content of treatment, evaluation and safety. In addition to recently published systematic reviews, additional literature reviews concerned assessment, safety and the dosage of exercise therapy. Related to the clinical questions, a framework for the therapeutic process and 12 draft recommendations were developed and discussed in the second meeting. In the third and last meeting the 12 recommendations regarding the delivery of physical therapy and exercise interventions were set and the level of agreement was determined.

Conclusions: The expert-meetings and literature searches led to 12 practice recommendations and a clear starting point for the development of the implementation strategy. Twelve practice recommendations regarding the delivery of physical therapy for patients with axSpA were developed, based on scientific evidence, expert opinion patient values. The field testing and development and execution of a dissemination and implementation strategy will be done in 2017 .

References:

[1] Dagfinrud $\mathrm{H}$, et al. Physiotherapy interventions for ankylosing spondylitis. Cochrane Database Syst Rev. 2008

[2] van der Heijde D, et al. 2016 update of the ASAS-EULAR management recommendations for axial spondyloarthritis. Ann Rheum Dis. 2017.

[3] Steeringgroup recommendations Spondyloartritis, Dutch Rheumatology Association, Guidelines for diagnosis and treatment of axial Spondyloartrtis, 2014.

[4] Van der Giesen et al. Use and content of physical and (group)exercise therapy for people with axial spondylarthritis, NVR congress, posterpresentation, 2016. Acknowledgements: The Dutch Arthritis Foundation financially supported this project.

Disclosure of Interest: None declared

DOI: 10.1136/annrheumdis-2017-eular.4432

\section{THU0749-HPR INCREASING PHYSICAL ACTIVITY IN PEOPLE WITH A CHRONIC DISEASE: EXAMINING THE EFFECTIVENESS OF A MOTIVATIONAL AND A PLANNING INTERVENTION, THEIR INTERACTION AND VARIOUS POTENTIAL MODERATORS AND MEDIATORS}

S. Hilberdink ${ }^{1}$, G.-J.Y. Peters ${ }^{2} .{ }^{1}$ Allied Healthcare Centre for Rheumatology and Rehabilitation, Groningen; ${ }^{2}$ Psychology and Education Science, Open University of the Netherlands, Heerlen, Netherlands

Background: Physical activity has many health benefits, especially in people with a chronic disease. Behaviour change interventions appear more effective than just advice when increasing physical activity. However, most studies that compare different interventions overlook interaction effects between methods and their parameters for effectiveness.

Objectives: Present study examined the effectiveness of a motivational and an action planning intervention for increasing physical activity in patients with a chronic disease. Their interaction as well as various potential moderators and mediators were studied in order to identify which intervention is effective in which patients.

Methods: In a healthcare centre specialised in the treatment of people with a chronic disease, participants whose physiotherapist would advise them to be more physically active were randomly assigned to one of four interventions in a factorial design: 1) a control condition with only advice, 2) a motivational intervention 
with information about health benefits and selecting personally relevant motives and social support sources regarding physical activity, 3) an action planning intervention in which participants specified which physical activities they would do, when, where and for how long and 4) a combination of these interventions. Prior to the intervention, participants' leisure time physical activity in the past week was measured with items from the SQUASH. Also behavioural intentions, expected social support and planning behaviour with regard to leisure time physical activity were measured. Directly after the intervention, positive outcome expectations and self-efficacy were measured. After a week, physical activity measures were administered again.

Results: 208 Participants $(71 \%$ female; mean age 62 years; $40 \%$ with a rheumatic disease) completed the study. Figure 1 displays the average change in physical activity for each intervention. Multiple regression analyses showed that both the motivational intervention $(\beta=0.22)$ and action planning $(\beta=0.31)$ significantly predicted a positive physical activity change and that these effects appeared additive as opposed to catalytic. The effects were not moderated by intention, expected social support or planning behaviour, nor mediated by outcome expectations or self-efficacy. The motivational intervention's effectiveness was weakly correlated with the number of personally relevant exercise motives identified $(r=0.22)$ and the action planning's effectiveness was weakly related to the number of plans made $(r=0.19)$ and to one's outcome expectations after the intervention $(r=0.21)$.

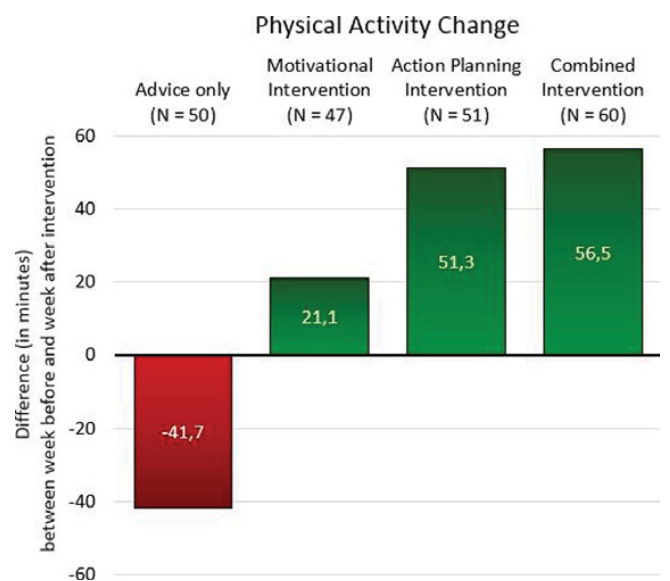

Conclusions: These data suggest that using a motivational or action planning intervention seems beneficial for increasing physical activity in patients with a chronic disease, independent of one's intentions, expected social support and planning behaviour. The data also suggest that action planning is preferable over a motivational intervention and that the added value of a motivational intervention is minimal if action planning is used.

This study was limited by only measuring physical activity after a week and by using self-report. Future studies should investigate longer term effects, other potential moderators and mediators and interaction effects between other promising behavioural change methods.

Disclosure of Interest: None declared

DOI: 10.1136/annrheumdis-2017-eular.2137

\section{THU0750-HPR THE EFFECTIVENESS OF THERAPEUTIC FOOTWEAR IN PATIENTS WITH RHEUMATOID ARTHRITIS: A SYSTEMATIC REVIEW AND META-ANALYSIS}

M. Tenten-Diepenmaat ${ }^{1}$, M. Leeden van $\operatorname{der}^{1,2}$, T. Vliet Vlieland $^{3}$, L.D. Roorda ${ }^{1}$, J. Dekker ${ }^{2,4} .{ }^{1}$ Amsterdam Rehabilitation Research Center, Reade; ${ }^{2}$ Department of Rehabilitation Medicine, VU University Medical Center, Amsterdam;

${ }^{3}$ Department of Orthopaedics, Rehabilitation and Physical Therapy, Leiden

University Medical Center, Leiden; ${ }^{4}$ Department of Epidemiology and

Biostatistics, Amsterdam Public Health research institute, VU University Medical Center, Amsterdam, Netherlands

Background: Therapeutic footwear is recommended in guidelines for the treatment of foot problems in patients with rheumatoid arthritis (RA) and commonly prescribed and frequently used, especially in patients with established RA, foot deformities or erosions in foot joints.

Objectives: The objectives of this study were to summarize the effectiveness of therapeutic footwear on foot function, pain, physical functioning, health-related quality of life, adherence, adverse events and patient satisfaction in patients with RA.

Methods: All randomized controlled trials, randomized controlled cross-over trials, (quasi-experimental) clinical trials, and uncontrolled studies investigating the effect of therapeutic footwear in patients with RA related foot problems were included. Therapeutic footwear consists of custom-made or ready-made shoes. A literature search was conducted in The Cochrane Central Registry for Controlled Trials (CENTRAL), PubMed, EMBASE and PEDro up to January 19, 2017. Selection and inclusion of articles, data extraction (using a standardized template) and assessment of methodological quality (using a checklist for between-group and within-group comparisons) was conducted by two independent reviewers. Quantitative data analyses was conducted, when quantitative data analysis was not possible qualitative data analysis was performed.

Results: Thirteen studies were identified. In five studies, one of which was of high quality, between-group differences were reported. In twelve studies, of which two were of high quality, within-group differences were reported. Qualitative datasyntheses for the within-group differences of custom-made therapeutic footwear resulted in weak evidence for the reduction of foot pain and improvement of physical functioning. Quantitative data-analyses of the within-group differences of ready-made therapeutic footwear resulted in a medium to large effect for the reduction of foot pain (SMD $-0.68,95 \% \mathrm{Cl}-1.00$ to $-0.37 ; \mathrm{P}=0.0001 ; 162$ participants), and a small to medium effect for the improvement of physical functioning (SMD $-0.32,95 \% \mathrm{Cl}-0.62$ to $-0.02 ; \mathrm{P}=0.04 ; 128$ participants). Qualitative data-analyses of the between-group differences of ready-made therapeutic footwear resulted in inconclusive evidence for foot pain and physical functioning.

Conclusions: In within-group designs, there is weak evidence for the reduction of foot pain and improvement of physical functioning after wearing custom-made therapeutic footwear. Furthermore, in within-group designs there is a medium to large effect of ready-made therapeutic footwear on the reduction of foot pain, and a small to medium effect on the improvement of physical function. Controlled, between-group designs resulted in inconclusive evidence. A definite RCT is necessary to investigate the between-group effectiveness of therapeutic footwear in patients with RA.

Disclosure of Interest: None declared

DOI: 10.1136/annrheumdis-2017-eular.5809

\section{THU0751-HPR EFFECTIVENESS OF FOOT ORTHOSIS IN PATIENTS WITH RHEUMATOID ARTHRITIS RELATED TO QUALITY OF LIFE AND PAIN. A SYSTEMATIC REVIEW AND META-ANALYSIS}

G. Gijon-Nogueron, L. Ramos-Petersen, S. Garcia-Mayor, J.M. Morales-Asencio. Nursing and Podiatry, University of Malaga, Malaga, Spain

Background: Foot pain and deformity is almost ubiquitous in RA and results in considerable physical and psychosocial impairment [1]. Epidemiological studies consistently suggest a $90 \%$ prevalence of foot pain despite advances in pharmacological therapy [2]. Mechanical and other non-pharmacological interventions such as orthoses and footwear, have an important role in managing foot pathology in patients with their systemic disease controlled $[1,3]$. The effectiveness of treatment with insoles, especially in early periods, was studied in a randomized controlled trial, which results suggested an immediate clinical improvement, reducing foot pain, disability and limited functionality.[1]

Objectives: The aim of this study is the effectiveness of foot orthosis in patients with rheumatoid arthritis in terms of quality of life and pain.

Methods: A systematic review and meta-analysis was conducted of randomized controlled trials.

Participants: Patients with rheumatoid arthritis were included. The criteria of exclusion were Juvenile Rheumatoid Arthritis, analysis of gait,

Intervention: Studies had to compare foot orthosis

Comparison: Other type of treatments, other type of foot orthosis, sham

Outcomes: Evaluation of Pain or Quality of life with any tool that measure this outcomes

The search was conducted in Cochrane, CINAHL, PubMed, EMBASE, LILACS, and Cuiden. An independent peer review was carried out. The Mesh term and fields used were foot, ankle, joint, rheumatoid arthritis, foot, orthosis, insole, foot orthosis.

Results: After the analysis of 71 studies, 4 were included for the systematic review. The 4 studies enrolled 285 participants. Follow-up periods varied from 6 to 30 months.

Only two studies were included in the meta-analysis [4,5], both of them with pain (measured with Foot Function Index) as the selected outcome.

A meta-analysis of the two trials showed that use of FO resulted in a nonsignificant improvement in disability compared with control (MD $(95 \% \mathrm{Cl}): 4.37$ $(-6.24,14.98) ; \mathrm{N}=64)$ (Figure 1).

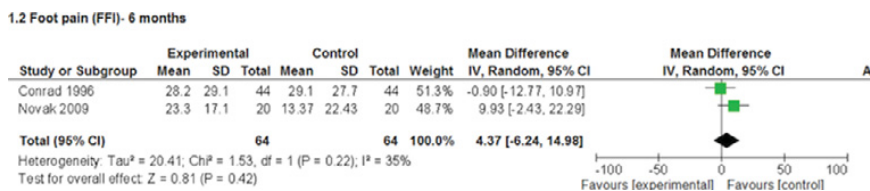

Conclusions: Foot orthoses showed improvements in pain and disability/quality of life, but no significant differences between groups were found.Future research needs to increase the number of RCTs in this topic because results are not conclusive.

\section{References:}

[1] Woodburn J, Barker S, Helliwell PS. A randomized controlled trial of foot orthoses in rheumatoid arthritis. J Rheumatol. 2002;29:1377-83.

[2] Otter SJ, Lucas K, Springett K, Moore A, Davies K, Young A, et al. Comparison 